\title{
Preface to the special issue: Continuity, Computability, Constructivity: From Logic to Algorithms 2014
}

\author{
ANDREJ BAUER \\ ULRICH BERGER \\ WILLEM FOUCHÉ \\ DIETER SPREEN \\ HIDEKI TSUIKI \\ MARTIN ZIEGLER
}

This issue of The Journal of Logic and Analysis includes selected papers submitted by participants of the Workshop "Continuity, Computability, Constructivity: From Logic to Algorithms", held in Ljubljana in the third week of September 2014.

The workshop was partially funded by the Faculty of Mathematics and Physics at the University of Ljubljana, the Institute for Mathematics, Physics and Mechanics, as well as the European Union. It was the fourth in a series of workshops aimed at bringing together researchers from exact real number computation, computable analysis, effective descriptive set theory, constructive analysis, and related fields. The overall objective is to apply logical methods in these disciplines to provide a sound foundation for obtaining exact and correct algorithms. At the same time, the conference was the third annual meeting of the COMPUTAL project, which is a research network between Europe, Russia, South Africa, and Japan funded by the European Union under the FP7-IRSES programme scheme.

A major motivation for the project collaboration is the fact that in safety-critical applications it is not sufficient to produce software that is only tested for correctness: its correctness has to be formally proven. This is true as well in the area of Scientific Computation. The problem here is that the current mainstream approach to numerical computing uses programming languages that do not possess a sound mathematical semantics. Hence, there is no way to provide formal correctness proofs.

The reason is that on the theoretical side one deals with well-developed analytical theories based on the non-constructive concept of a real number. Implementations, on the other hand, use floating-point realisations of real numbers which do not have a 
well-studied mathematical structure. Ways to get out of these problems are promoted under the slogan "Computing with Exact Real Numbers". Well developed practical and theoretical bases for exact real number computation and, more generally, computable analysis are provided by Scott's Domain Theory and Weihrauch's Type Two Theory of Effectivity. In both theories real numbers and similar ideal objects are represented by infinite streams of finite objects. They can locally be manipulated by Turing machines. In contrast to the theory of computing with finite data, the study of computing with infinite data crucially depends on topological considerations.

A related approach is pursued in constructive analysis by using intuitionistic logic. As is well known, proofs of existential statements in this logic allow the extraction of algorithms computing the object that is stated to exist. By their nature these algorithms are correct.

Many important problems are not only hard to compute, they are even not computable at all. To measure the degree on non-computability one relates their difficulty to the difficulty of certain well-known master problems by using reducibility notions that generalise the classical many-one reducibility.

The present issue contains contributions to all these areas.

As usual, there are many people to be thanked. This is in particular true for the organizing committee of the Ljubljana meeting. They did a wonderful job. Moreover, we want to thank the referees for having taken the burden of carefully reading and commenting the submissions. Last, but not least, we are very grateful to the Editor-in-Chief of The Journal of Logic and Analysis for the opportunity to publish in this issue of the journal.

The papers have undergone a rigorous reviewing process in accordance with the standards set by The Journal of Logic and Analysis. Submissions by special issue editors were handled by others so as to protect the anonymity of the reviewers and to avoid conflicts of interest.

Special issue editors:

Andrej Bauer

Ulrich Berger

Willem Fouché

Dieter Spreen

Hideki Tsuiki

Martin Ziegler

Fakulteta za matematiko in fiziko, Univerza $v$ Ljubljani

Jadranska 21, 1000 Ljubljana, Slovenia

Journal of Logic \& Analysis 9:c0 (2017) 
Department of Computer Science, Swansea University Singleton Park, Swansea SA2 8PP, UK

Department of Decision Sciences, University of South Africa PO Box 392, UNISA 0003, South Africa

Department of Mathematics, University of Siegen 57068 Siegen, Germany

Graduate School of Human and Environmental Studies, Kyoto University Yoshida-nihonmatsu-cho, Sakyo-ku, Kyoto 606-8501, Japan

School of Computing, KAIST

291 Daehak-ro, Yuseong-gu, Daejeon 34141, Republic of Korea

Andrej.Bauer@andrej.com, u.berger@swansea.ac.uk, fouchwl@gmail.com, spreen@math.uni-siegen.de, tsuiki@i.h.kyoto-u.ac.jp, ziegler@cs.kaist.ac.kr

http://andrej.com, http://www-compsci.swan.ac.uk/ csulrich/, http://www-compsci.swan.ac.uk/ csulrich/, http://www.uni-siegen.de/fb6/tcs/team/spreen/, http://www.i.h.kyoto-u.ac.jp/ tsuiki/index-e.html, https://cs.kaist.ac.kr/people/view?idx=517\&kind=faculty

Received: 14 March 2017 